\title{
GeoMAC: Geo-Backoff based Co-operative MAC for V2V networks
}

\author{
Sanjit Kaul and Marco Gruteser \\ WINLAB, Rutgers University \\ NJ, U.S.A \\ \{sanjit, gruteser\}@winlab.rutgers.edu
}

\author{
Ryokichi Onishi and Rama Vuyyuru \\ TOYOTA InfoTechnology Center \\ NJ, U.S.A \\ \{onishi, rama\}@toyota-itc.com
}

\begin{abstract}
This paper presents a preliminary design of GeoMAC, a MAC protocol that exploits spatial diversity in highly mobile wireless networks. It aims to achieve low latency and high reliability, goals that are intrinsic to the success of many envisioned vehicular safety applications. Conventional MAC layers address reliability through ARQ mechanisms that retransmit messages from the source, if earlier transmissions were not acknowledged. These schemes essentially exploit temporal diversity since retransmissions are only likely to succeed if the channel has improved. GeoMAC exploits spatial diversity, by allowing other nearby nodes to opportunistically forward and retransmit messages. Through a geo-backoff mechanism it uses geographic distance to the destination as a heuristic to select the forwarder most likely to succeed. This mechanism does not require nodes to monitor channel state or position of their neighbors, except for approximate node density, thus enabling their use in highly mobile networks with low coordination overhead. The performance of GeoMAC is evaluated via tracedriven ns2 simulation using packet error measurements from a freeway environment. GeoMac leads to lower delay jitter combined with up to $50 \%$ packet delivery rate gains, compared to the AODV and GPSR routing protocols, which also take advantage of nearby nodes for packet forwarding. Spatial diversity is also shown to better utilize available channel opportunities than ARQ mechanisms.
\end{abstract}

\section{INTRODUCTION}

Vehicle-to-vehicle communication enables safety assistance, traffic improvement, customer service and infotainment applications. This paper focuses on safety assistance applications, such as an extended electronic brake light or intersection collision avoidance, which promise to reduce vehicle accidents by transmitting warning messages to notify cars and their drivers of dangerous situations. To be effective, these applications require low latency and highly reliable vehicle-to-vehicle communication protocols.

High mobility results in a highly time-varying wireless channel [1]. Sample RSSI measurements obtained with 802.11a nodes on a freeway-like stretch of US-1, with a moving transmitter and stationary receiver besides the road are shown in Fig. 1(a). Measurements show up to $10 \mathrm{~dB}$ RSSI changes every few hundred milliseconds likely due to shadowing from obstructions. Note that the observed temporal coherence in fading is about $100 \mathrm{~ms}$ during dips in signal, which would lead to significant delays for protocols solely based on ARQ schemes such as Stop-and-Wait [2] . If a packet is lost due to a reduction in received signal strength, the following MAC retries are also likely to fail until the signal strength has recovered. Ad hoc routing protocols, can also increase reliability by forwarding the message over an alternate path. Protocols such as AODV [3] or DSR [4], however, can efficiently adjust routes only on larger time scales-they incur significant route discovery overhead when routes change every $100 \mathrm{~ms}$.

This work addresses the challenge of reliable communication in a highly time-varying channel through the GeoMAC protocol, which exploits the broadcast nature of the wireless medium to achieve spatial diversity. It uses receiver diversity, meaning that any of the nodes that can decode a transmitted message can opportunistically forward it towards the destination. Forwarders are selected in distributed manner via a geographic backoff (geobackoff), that assigns the highest priority to nodes with the smallest Euclidean distance to the destination node. This allows forwarding with only an approximate knowledge of node density, no detailed monitoring of channel state or neighbor positions is necessary. It assumes, however, that nodes know their own position, for example through the Global Positioning System. If a transmission fails to achieve any forward progress (i.e., reach a node closer to the destination), the protocol can use cooperative ARQ by opportunistically retransmitting messages from the next closest node, rather than the original forwarder. Opportunistically choosing different forwarders and retransmitters increases spatial diversity and can circumvent delays imposed by temporal coherence of the channel.

The remainder of this paper is organized as follows. In Sec II we detail the protocol's design and follow it with implementation in Sec III. Sec IV describes the trace-driven simulation setup to evaluate spatial diversity gains in a vehicular scenario and compare the GeoMAC protocol with standard routing protocols. The results in $\mathrm{Sec} \mathrm{V}$ show that spatial diversity can provide additional gains over temporal diversity and that the GeoMAC protocol reduces delays especially with regard to outliers. Related work is discussed in Sec VI. We conclude with a summary of results and future work in Sec VII.

\section{GeO-COOPERATIVE MAC}

The key challenge to exploiting spatial diversity in highly mobile networks is the efficient selection of the next transmitter, if direct transmission to the destination failed. This distributed decision making is enabled via a geobackoff, in which the backoff interval increases with a node's Euclidean distance from the destination. The sender includes the destination node's geographic position in the packet header, which allows each node to calculate its backoff interval independently. This assumes that each car monitors its own position (e.g., via the Global Positioning System). It also 

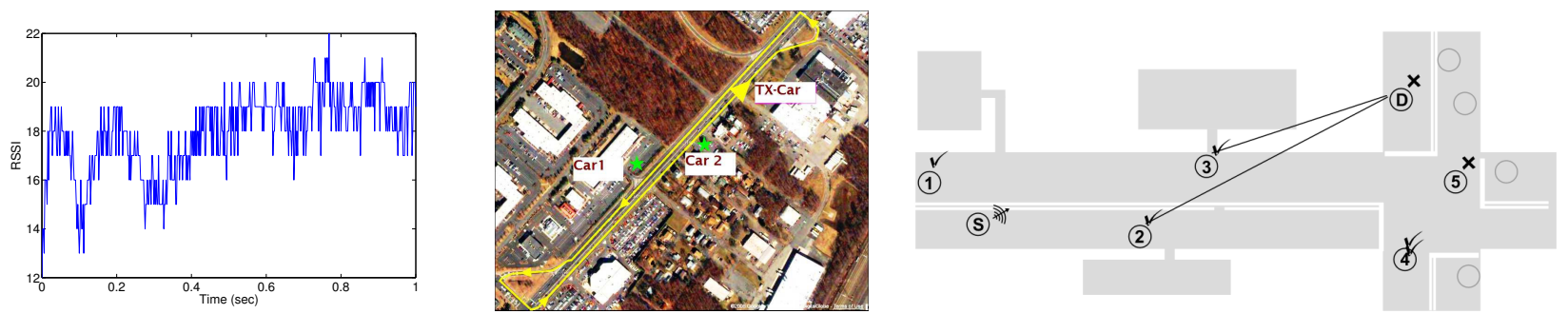

(a) Sample SNR fluctuations on vehicle-to- (b) US-I, two receivers and a trans- (c) Source (S) sends message for destination (D). Radios 1, 2 vehicle communication link in freeway environ- mitter, RSSI and PER measurement and 3 are prospective forwarders. ment setup

Fig. 1. Vehicular Channel Measurements and Setup

assumes that vehicles share these coordinates with potential communication partners, for example via periodic beacons. For an example of geographic forwarder selection, consider Figure 1(c), where the source (S) transmits a message that is received by nodes (1), (2) and (3) but not by the intended destination (D). Node (3) will calculate the shortest geobackoff and first forward the message, because it is closest to the destination position. Beyond this opportunistic forwarding, spatial diversity is also exploited by co-operative ARQ wherein (2) would retransmit the message if node (3)'s forwarding fails. The nature of this back-off scheme allows additional nodes to dynamically participate in forwarding whenever they have overheard a transmission, no explicit joining procedure or route setup is necessary.

\section{A. Protocol Timing}

Figure 2 shows GeoMAC's timing diagram for the same example ${ }^{1}$. When nodes $1-3$ receive the packet, they start their geobackoff timers. On expiration of node 3's timer the node forwards the DATA frame. On receipt of the first bit of the DATA frame, the co-operating forwarders 1 and 2 suspend their back-off timers for the transmission duration and an additional VACK-TIMEOUT. If no VACK is received after the VACK-TIMEOUT, the forwarders resume their backoff timers (a variation where forwarders restart timers is also possible). Now node 4 , which has successfully overheard the transmission from node 3 , transmits the message that is acknowledged by a VACK from the destination.

\section{B. Geobackoff}

The geobackoff must efficiently map geographic distance to backoff intervals, which minimize the chance for collisions without imposing unnecessary delays. Assume that the minimum time-granularity that can be supported at the Physical layer is the slot time $t_{s}$. Further, let $d_{n} \mathrm{~m}$ be the distance of a cooperative forwarder node $n$ from the destination. The node $n$ then sets its backoff timer to $\left\lceil\frac{d_{n}}{\delta}\right\rceil t_{s}$, which is rounded to the next integral multiple of $t_{s}$, where $\delta$ is the expected minimum spatial separation between any two forwarders given the current vehicle density (e.g., for a very congested single lane road a $\delta$ of $5 \mathrm{~m}$ may be reasonable).

\footnotetext{
${ }^{1} \mathrm{~A}$ more complete discussion of issues involved and probable solutions is in Section VII
}

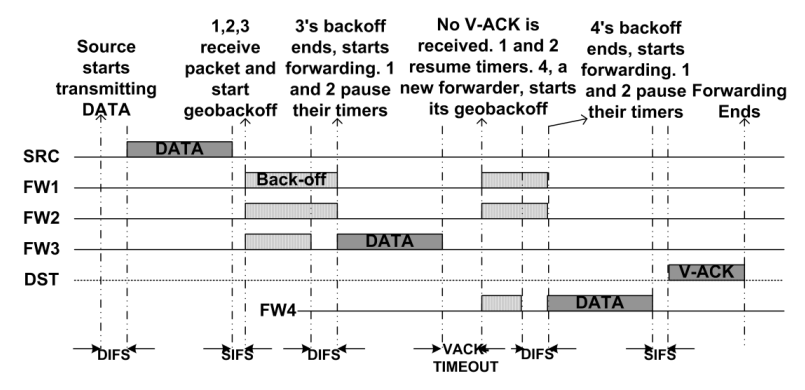

Fig. 2. Protocol Timing assuming back-off is resumed after VACKTIMEOUT

$\delta$ could be estimated based on overhearing periodic beacon transmissions ${ }^{2}$.

\section{Protocol Implementation}

GeoMAC is implemented as a Click [5] router element, which can be used in the ns2 [6] network simulator through the nsclick [7] framework. ${ }^{3}$. For ease of prototyping, GeoMAC is based on parts of the ns2 802.11 MAC implementation-all packets are transmitted using 802.11 MAC broadcast (hence 802.11 MAC retries are disabled).

To be able to suspend backoff while other transmissions are underway, the implementation monitors the 802.11 MAC state. Since the GeoMAC is implemented in the click framework, while packet transmissions are simulated through ns2, the implementation needs to account for additional processing times used for communicating state between click and ns2. In particular if the MAC is in a receiving state, GeoMAC must defer any current transmissions by this PROCESSING_TIME. We empirically determined this time to transfer a message received in ns 2 to GeoMAC to be 330us.

The timeslot is set to, $t_{s}=$ DIFS + PROCESSING_TIME ${ }^{4}$. VACK_TIMEOUT includes the

\footnotetext{
${ }^{2}$ We ignore the difference in propagation delay to different forwarders as for typical PHY implementations, the slot time $t_{s}$ is much greater than the propagation delay even at the transmission powers considered in vehicular networks.

${ }^{3}$ We chose a click implementation because it will later allow us to run it in an actual Linux system, say emulated over 802.11 implementation

${ }^{4}$ The large timeslot value is because GeoMAC currently interfaces with PHY via 802.11
} 
processing time needed at the destination to start sending a VACK and an additional amount to account for the 802.11 MAC DIFS, yielding DIFS + PROCESSING_TIME - Assuming negligible propagation time, the first bit of a VACK must reach the waiting forwarders within a VACK_TIMEOUT. DIFS is set to 40us.

Re-transmission of a packet by a forwarding node is supported, in case spatial diversity fails to forward a packet ${ }^{5}$. A forwarder sets its re-transmission timer if no VACK is received from DST after itself and other forwarders closer to DST have forwarded the packet at least once. The retransmission timer is currently set to VACK_TIMEOUT, a forwarder waits for one more forwarder to forward a message before resorting to re-transmission. Re-transmissions are currently not implemented at SRC.

\section{EVALUATION FRAMEWORK}

To study feasibility of the GeoMAC scheme, the evaluation compares GeoMAC's performance with the AODV and GPSR routing protocols on the IEEE 802.11 MAC in terms of delay, jitter, and packet delivery rate using tracedriven ns2 simulations. We also first evaluate achievable spatial diversity gains in our simulations scenario by comparing three basic forwarding schemes without routing overhead. In scheme fixed-forwarder-retransmit (FixFWReTX), a packet is retransmitted by SRC until successfully received by a constant pre-selected forwarder, emulating the standard 802.11 MAC. In scheme blind-forwarder-selectionretransmit (BlindReTX), the destination is switched between the two forwarders before each retransmission of a packet. Switches occur blindly without any knowledge of channel state. Finally, the Spatial Diversity scheme broadcasts each (re-)transmission to both forwarders.

AODV is an on-demand routing protocol that looks for a route, when one to a packet destination is unknown. Routes over which no data is transmitted over a period of time are expired. It maintains only a single route to a destination. GPSR uses the position of nodes to make forwarding decisions. It uses greedy forwarding (maximize spatial progress) and perimeter mode when there are no nodes, closer to destination, in range. 802.11 MAC level retry failures and neighbor expiry timers are used to remove nodes from neighbor lists. It may maintain more than one route to a destination.

\section{A. Simulation Setup}

The simulations consider a four node linear topology, in $\mathrm{a} \approx 400 \mathrm{~ms}$ snapshot scenario where significant changes in channel state occur but geographic movement of the nodes is small $(<15 \mathrm{~m})$ compared to the node density. We therefore keep the node positions that GeoMAC observes stationary, at relative positions $(0,0)$ for the source $(\mathrm{SRC}),(0,100)$ for forwarder 1 (FW1), (0,200) for forwarder 2 (FW2), and $(0,300)$ for the destination (DST). We configure the

\footnotetext{
${ }^{5}$ We use only two forwarders for GeoMAC evaluation and so allow for re-transmission. Its requirement in vehicular networks, where we typically will have more than just two forwarders, needs evaluation
}

GeoMAC parameter $\delta$ to $100 m$, the optimal value for this configuration. Further, the simulations use a $1 \mathrm{Mbps}$ PHY rate $^{6}, 802.11$ MAC with default parameters $(\mathrm{CWMIN}=31$, CWMAX =1024) and without RTS/CTS. The data traffic is CBR at 10 packets/sec. A total of 4990, 512 byte packets are sent during a simulation $\operatorname{run}(\approx 1200$ repetitions of the snapshot).

Channels are emulated by replaying traces from an automotive experiment that measured packet reception rates on a freeway-like segment of U.S. Route 1. Each vehicle carried a roof-mounted, $7 \mathrm{dBi}$ dipole, antenna connected to Atheros 5212, 802.11a radios. A moving vehicle (TX-Car), traversing a path, shown by a yellow line in Fig 1(b), of the US-1 freeway, passed two stationary vehicles (Car1 and Car2 in figure) parked in lots adjacent to the road on opposite sides while broadcasting 500 packets per second. The stationary vehicles record traces of packet reception. To evaluate spatial diversity gains possible under favorable conditions, we selected $\mathrm{a} \approx 400 \mathrm{~ms}$ snapshot, representative of regions in the trace where both vehicles receive packets, but channel to neither is very reliable (for the snapshot the packet error rates are $58.71 \%$ and $45.77 \%$ ). Figure 3(a) shows the selected snapshot. Each dot in the time-series corresponds to a received packet. As the table indicates, out of the total 201 transmitted, 53 packets were not received by both vehicles. 168 packets ( $\approx 76 \%$ of total transmission) were received by at least one vehicle.

We modified ns2 to interpret these packet traces instead of using one of the default propagation models. The trace is played at the rate of reading a new packet, i.e., a new channel condition, every $2 \mathrm{msec}$. If the packet in the trace was received the channel indicator is set to receive, otherwise to drop. Whenever a packet is transmitted in the simulation scenario, it will check the current channel indicator for the channel between source of the packet and each possible receiver to determine which nodes should receive the packet. The trace is repeated as many times as required over a simulation time.

The four node topology is subjected to two different sets of channel assumptions, one where the lossy channels lie between the forwarders and the destination and one where the lossy channels lie between the source and the forwarders. The forwarders always have perfect channels between each other and SRC and DST are outside each other's communication range.

- TXDiv: The channel between FW1 and DST is emulated by the trace Car1 in Figure 3(a). Car2 emulates the channel between the FW2 and DST. Channels between the SRC and the forwarders are assumed perfect. A packet always arrives at both forwarders but may not be received at the destination after forwarding, allowing evaluation of transmit diversity (TXDiv) to the DST.

- RXDiv: The channels between the SRC and forwarders are emulated by the traces Carl and Car2 while the

\footnotetext{
${ }^{6}$ affects only the packet transmission time as the channel is emulated by traces as explained later
} 
channels between the forwarders and the DST are assumed perfect. The scheme allows evaluation of receiver diversity (RXDiv) for transmissions from the SRC.

\section{Results}

\section{A. Spatial vs Temporal diversity}

We first demonstrate the gains that can be obtained by exploiting spatial diversity over two other prototype schemes in the RXDiv case ${ }^{7}$. Using the described simulation setup, we measure the number of transmissions (i.e., first transmission of a packet plus all retransmissions from same or other nodes) necessary to successfully deliver the packet to one of the forwarders.

Figure 3(b) shows the cumulative distribution function of the number of transmissions necessary for successful delivery of each packet under three basic forwarding schemes. Note that for scheme FixFWReTX we include a curve for each forwarder, because the scheme does not automatically use both, and performance is very dependent on the chosen forwarder. When the pre-selected forwarder is FW1, less than $40 \%$ of packets are received successfully on first attempt. For FW2, around $55 \%$ of packets are delivered by a single transmission. More than $20 \%$ and $8 \%$ of the packets need more than 4 transmissions and the maximum number of transmissions is $>16$ (simulations were limited to max 15 re-transmissions) and 10 for FW1 and FW2, respectively. Scheme BlindReTX closely tracks the performance of the better forwarder under scheme FixFWReTX, its switching strategy automatically settles on the better forwarder to use without the need for it to be pre-configured. The spatial diversity scheme, however, improves packet reception rate further, to $75 \%$ and $90 \%$ with just one and two transmissions, respectively. This represents a gain of two retransmissions over scheme FixFWReTX using the better FW2 and a gain of 6 transmissions over scheme FixFWReTX using FW1. The maximum transmissions that any packet requires is 6 compared to 10 or $>16$ for scheme FixFWReTX.

This result indicates that potential exists in vehicular communications for exploiting spatial reuse, especially with receive diversity. It can significantly improve delay for a given reliability and vice-versa, in certain vehicular communication situations.

\section{B. GeoMAC vs AODV and GPSR}

The evaluation presented above, ignores the requirement of a protocol to gather information about available forwarders, forwarder selection and last but not the least, coordination between the forwarders(to avoid collisions and multiple forwardings of a packet). We next compare the ns-2 implementations of our proposed protocol GeoMAC, AODV and GPSR, that account for this protocol overhead for TXDiv and RXDiv.

\footnotetext{
${ }^{7}$ The gains for TXDiv are similar and are omitted for brevity
}

1) Packet Delivery Rate: Routing overhead is very sensitive to the maximum number of retransmissions configured in the 802.11 MAC, since fewer frame retransmissions lead to more route change overhead due to more packet errors reaching the routing layer. We therefore compare results dependent on the total number of transmissions permitted, which for AODV and GPSR corresponds to the number of MAC transmissions of a packet by a forwarder. However, it puts no limit on the number of times a packet is re-routed by SRC (for example GPSR may try to re-route a packet via another neighbor which amounts to multiple transmissions of a packet by SRC). Since GeoMAC also includes cooperative ARQ, we define the number of transmissions as the maximum cumulative number of transmissions over all forwarders. Since the topology contains two forwarders, 2 is the minimum transmission limit for GeoMAC. As another example, a value of 4 corresponds to the default 2 possible forwardings and one more forwarding by each of the forwarders.

Figure 3(c) shows packet reception rate (out of 4990 transmitted) for different maximum number of transmissions settings (NumTrans) in the TXDiv case. It shows sizeable gains from exploiting only spatial diversity in GeoMAC, $($ NumTrans $=2)$. GeoMAC delivers $71 \%$ of the packets as against $\approx 10 \%$ by AODV and GPSR for NumTrans $=1$ and $45 \%$ by AODV and $20 \%$ by GPSR for NumTrans $=2$. AODV does much better than GPSR, especially for a smaller NumTrans, because it adjusts to route breaks, due to a bad channel to a currently selected forwarder, more dynamically than GPSR $^{8}$. As expected packet delivery rate increases with higher NumTrans, which reduces route changes and puts the onus of packet delivery on the 802.11 MAC which allows exploitation of temporal diversity in the channel. GeoMAC achieves $100 \%$ packet delivery at NumTrans $=8$. AODV comes close to $100 \%$ at NumTrans $=12$. GPSR performs slightly worse than AODV.

Note that the routing protocols with NumTrans limited to 1 fall significantly short compared to the packet delivery rate the basic scheme FixFWReTX achieved without any retransmissions. We attribute this to routing overhead.

2) Receiver Diversity: For RXDiv, the lack of a retransmission mechanism at SRC in the current implementation of GeoMAC, restricts its NumTrans to a value of 2.9 The efficacy of using spatial diversity is further brought out by packet reception gains of $40 \%$ at NumTrans $=2$ for GeoMAC (not shown), over AODV and GPSR.

3) Delay Performance: Figure 3(d) plots the average delays for packets successfully received by DST, for TXDiv. Figure 3(e) plots the corresponding jitter, which is measured as the standard deviation of the delay. The delay for AODV and GPSR is split into routing-related $(\mathrm{Rt})$ and non-routing related (Non-Rt) (802.11 MAC back-off, retry and packet

\footnotetext{
${ }^{8}$ For the selected GPSR beacon rate of once per second

${ }^{9}$ Under RXDiv , GeoMAC will be able to deliver a packet as long as at least one FWD has a good channel to the SRC, else the packet will be dropped. The retransmission mechanism at the forwarders won't be used as the channels from them to the DST are perfect.
} 


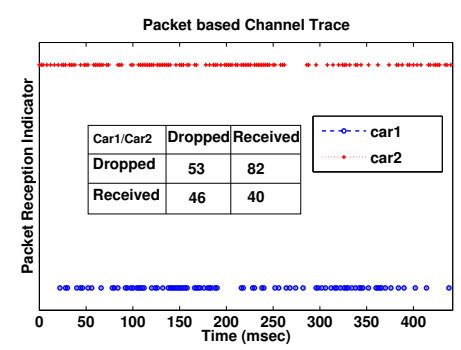

(a) PER Trace used for simulation.

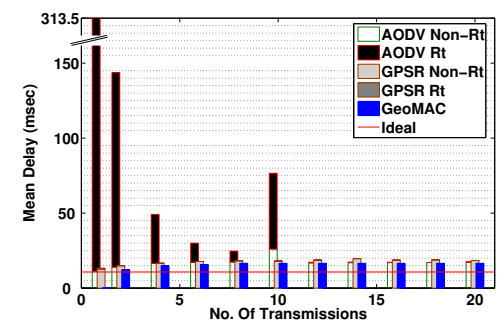

(d) Packet Transmission Delay (TXDiv). Note that the $y$-axis is omitted from 160-300 for plot clarity

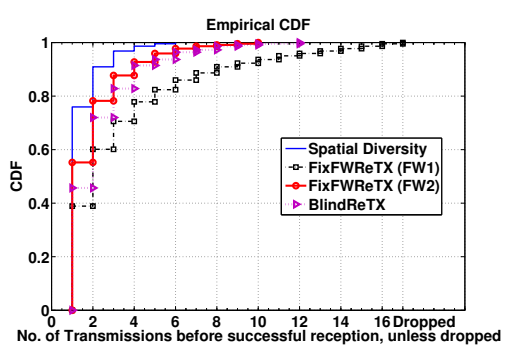

(b) Spatial vs. Temporal Diversity gains

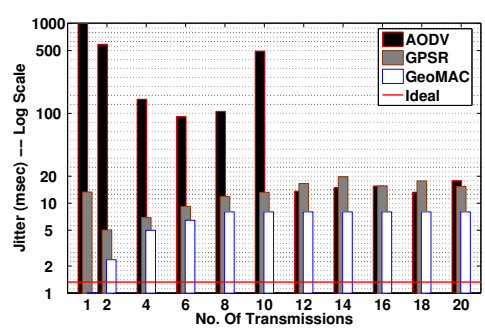

(e) Packet Transmission Jitter (TXDiv)

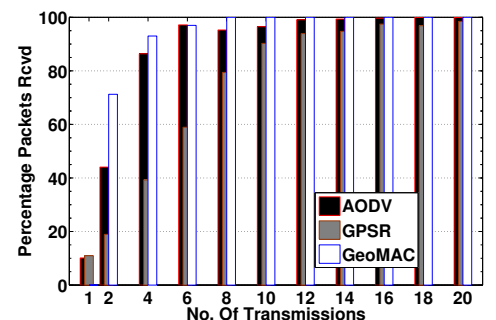

(c) Number of Packets Received (TXDiv)

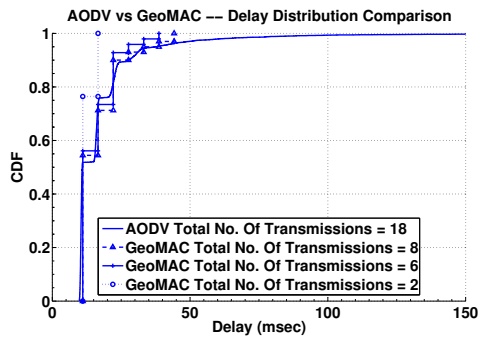

(f) AODV delay CDF vs. GeoMAC (TXDiv)

Fig. 3. Spatial Diversity and GeoMAC evaluation.

transmission and propagation). GeoMAC has no routing overheads and the delays incurred are due to the Geobased back-off, VACK_TIMEOUT, retransmission timers and transmission and propagation.

Figures 3(d) and 3(e) contain a red line which marks the mean delay and jitter assuming an ideal forwarding MAC. This ideal mechanism exploits all available good channel opportunities via either FW1 or FW2. In case of TXDiv, it knows channels between forwarders and the DST in advance and can schedule a transmission via a forwarder to ensure that the DST receives the packet at the earliest possible good channel opportunity. The only delays suffered, other than waiting for a good channel opportunity, are transmission and propagation delays. For TXDiv, the mean delay and jitter for the ideal MAC are $10.7288 \mathrm{msec}$ and $1.2279 \mathrm{msec}$, respectively.

AODV leads to a maximum delay of $313.8 \mathrm{msec}$ for NumTrans $=1$ and a minimum delay of $17.1 \mathrm{msec}$ for NumTrans $=18$. For smaller NumTrans, the large delays are routing related, $96.5 \%$ for NumTrans $=1$ and $42 \%$ for NumTrans $=6$. The jitter values, Figure 3(e), are considerably higher for NumTrans $<=10$.

Increased NumTrans leads to less routing delay and jitter, as channel conditions that would have lead to breaks in routes (and hence additional routing requests) are now alleviated by MAC retries. The re-routing overheads, seen for small NumTrans, also correspond to low packet delivery rates (Figure 3(c)). Dynamic routing by AODV seems incapable of adjusting routes fast enough to keep track of changing channel conditions.

GPSR, unlike AODV, doesn't look for routes dynamically. It routes via neighboring nodes, whose information it has via beacon packets that are exchanged periodically. Lack of dynamic routing leads to less packets delivered for the selected beacon rate as seen earlier. However, it also leads to less routing, small delay and jitter on the delivered packets. GeoMAC precludes the need for dynamic routing by exploiting spatial diversity. It keeps delay and jitter small by incorporating forwarder selection at the MAC layer.

Figure 3(f) compares the jitter performance of AODV for minimum mean delay and GeoMAC for TXDiv for NumTrans $=2,6,8$. AODV, even at 18 NumTrans for which AODV's mean delay is the smallest, has a much larger spread in delay than GeoMAC. For NumTrans $=8$ (where all packets are successfully delivered) the maximum delays are $44.2 \mathrm{msec}$, while with AODV (at 18 NumTrans) 144 packets $(\approx 3 \%$ of received) are received with delays greater than $50 \mathrm{msecs}$ and the maximum observed delay is $300 \mathrm{msec}^{10}$. For smaller NumTrans of 2,4, or 6 AODV requires more than $50 \mathrm{~ms}$ for $24.48 \%, 11.69 \%$ and $6.21 \%$ of packets, respectively.

The delay and jitter performance for RXDiv are similar to TXDiv for AODV and GeoMAC. GPSR, however, shows increase in jitter with increasing NumTrans, which is because in RXDiv the channels between SRC and the forwarders, unlike in TXDiv, are lossy.

Overall, GeoMAC achieves high packet delivery rates with the lowest delay. In particular, its delay shows significantly fewer outliers than by adding spatial diversity at the routing layer.

\section{RELATED WORK}

The use of geographic position for routing was first suggested in the 1980s [8] for stationary packet radio networks.

\footnotetext{
${ }^{10}$ Figure 3(f) plots the AODV CDF for values up to $160 \mathrm{msec}$ only
} 
Imielinski and colleagues pioneered location as an addressing mechanism for the mobile Internet [9]. Research has progressed towards using geographic information in routing for wireless ad hoc and sensor networks [10]-[15]. Similarly multicast protocols that serve all nodes in a geographic region [16], [17] have been developed. These protocols focus more on sparse scenarios with routing around voids in the communication network as a main challenge. In [18] the authors propose the ExOR protocol which uses opportunistic routing under which paths are chosen dynamically on a per path basis. In [19] a contention based forwarding scheme is proposed. It selects the next hop through a distributed contention process based on the actual geographic position of each node. The mechanism bears similarities to the geobackoff part of our protocol but is implemented above the MAC layer and the schemes differ in their goal. Whereas contention-based forwarding seeks to reduce or eliminate location beacon messages, GeoMAC aims at exploiting spatial diversity for increased reliability. In [20] the authors propose position and map based forwarding and geocasting for vehicular networks. Gains are obtained in the highly mobile vehicular scenarios as no route creation is required. In our work we use location to carry out position based forwarding and also to exploit spatial diversity, enabling packet forwarding with minimum delays and high delivery probability.

\section{CONCLUSIONS}

We presented GeoMAC, a MAC-based protocol that exploits spatial diversity, inherent in a vehicular channel. Forwarder selection for transmission over the next hop is enabled in a distributed manner via geobackoff, which selects forwarders in decreasing order of spatial progress. It also uses a cooperative ARQ mechanism. We conclude that

- Spatial diversity with just two forwarders (NumTrans $=2$ for GeoMAC) can provide packet delivery of $20 \%$ on first transmission and of 25-50\% when considering full AODV and GPSR routing overheads ( with two 802.11 MAC retries).

- GeoMAC lead to minimal mean delays and very low jitter in comparison to AODV. Mean AODV delays for $1 \leq$ NumTrans $\leq 8$ range from $24.7-300 \mathrm{msec}$ as against a mean of $12.4-16.5 \mathrm{msec}$ for GeoMAC (all NumTrans values). Further, AODV shows jitter values of 92.1$1000 \mathrm{msec}$ as against a jitter of 2.4-8msec for GeoMAC. The high jitter and delay values are due to routing overheads under fast changing channel conditions.

- In general, both AODV and GPSR show better packet delivery, delay and jitter performance as number of 802.11 MAC retries increases, putting the onus of packet delivery on the MAC layer. We show that a MAC exploiting spatial diversity performs better than a retrybased MAC, which transmits over the best available channel.

The GeoMAC spatial diversity gains are likely to improve further, if more than two forwarders are available, which is likely in many vehicular network scenarios.

\section{A. Future Work}

This preliminary protocol design raises several questions for future work. First, the protocol must adapt the value of the $\delta$ parameter to vehicle density. The optimal value could be derived from neighbor information collected via periodic beacons or from overheard data packets. Second, it must handle concurrent transmission between multiple sourcedestination pairs. The current design may lead to too many collisions. This could be achieved by incorporating a random component into backoff choices. A single forwarder may also have to keep track of multiple geobackoff timers. Finally, the protocol should suppress unnecessary transmissions if multiple forwarders are not within range of each other. The current protocol may lead to redundant or colliding transmissions. One approach may be specifying a forwarding region, to exclude extraneous nodes outside this region from the forwarding process. It could also restrict the number of co-operative transmissions of a DATA packet.

\section{REFERENCES}

[1] M. Torrent-Morreno, M. Killat, and H. Hartenstein, "The challenges of robust inter-vehicle communications," in Vehicular Technology Conference, VTC-2005-Fall. IEEE, Sept., 2005, pp. 319-323.

[2] Bertsekas and Gallager, Data Networks. Prentice Hall, 1991.

[3] C. E. Perkins and E. M. Royer, "Ad-hoc on-demand distance vector routing," in Proceedings of the 2nd IEEE Workshop on Mobile Computing Systems and Applications, New Orleans, LA., February 1999, pp. $90-100$.

[4] D. Johnson, D. Maltz, and J. Broch, DSR: The Dynamic Source Routing Protocol for Multihop Wireless Ad Hoc Networks. AddisonWesley, 2001, ch. 5, pp. 139-172.

[5] E. Kohler, R. Morris, B. Chen, J. Jannotti, and M. F. Kaashoek, "The click modular router," ACM Transactions on Computer Systems, vol. 18 , no. 3 , pp. 263-297, Aug 2000.

[6] I. S. Institute, "The Network Simulator - ns-2," http://www.isi.edu/ nsnam/ns/.

[7] M. Neufeld, A. Jain, and D. Grunwald, "Nsclick:: bridging network simulation and deployment," in Proceedings of the 5th ACM international workshop on Modeling analysis and simulation of wireless and mobile systems. ACM Press, 2002, pp. 74-81.

[8] H. Takagi and L. Kleinrock, "Optimal transmission ranges for randomly distributed packet radio terminals," IEEE Transactions on Communications, vol. 32, no. 3, pp. 246-257, 1984.

[9] J. C. Navas and T. Imielinski, "Geocast: geographic addressing and routing," in MobiCom '97. New York, NY, USA: ACM Press, 1997, pp. 66-76.

[10] B. Karp and H. T. Kung, "Gpsr: greedy perimeter stateless routing for wireless networks," in MobiCom '00. New York, NY, USA: ACM Press, 2000, pp. 243-254.

[11] A. Rao, C. Papadimitriou, S. Shenker, and I. Stoica, "Geographic routing without location information," in MobiCom '03. New York, NY, USA: ACM Press, 2003, pp. 96-108.

[12] S. Basagni, I. Chlamtac, V. R. Syrotiuk, and B. A. Woodward, "A distance routing effect algorithm for mobility (dream)," in MobiCom '98. New York, NY, USA: ACM Press, 1998, pp. 76-84.

[13] Y.-B. Ko and N. H. Vaidya, "Location-aided routing (LAR) in mobile ad hoc networks," Wireless Networking, vol. 6, no. 4, pp. 307-321, 2000.

[14] G. Xing, C. Lu, R. Pless, and Q. Huang, "On „greedy geographic routing algorithms in sensing-covered networks," in MobiHoc '04. New York, NY, USA: ACM Press, 2004, pp. 31-42.

[15] Y.-J. Kim, R. Govindan, B. Karp, and S. Shenker, "On the pitfalls of geographic face routing," in DIALM-POMC '05. New York, NY, USA: ACM Press, 2005, pp. 34-43.

[16] Q. Huang, C. Lu, and G.-C. Roman, "Spatiotemporal multicast in sensor networks," in SenSys '03. ACM Press, 2003, pp. 205-217.

[17] Q. Huang, C. Lu, and G. Roman, "Mobicast: Just-in-time multicast for sensor networks under spatiotemporal constraints," 2002. [Online]. Available: citeseer.csail.mit.edu/article/huang02mobicast.html

[18] S. Biswas and R. Morris, "Opportunistic routing in multi-hop wireless networks," in In Proceedings of HotNets 2005, 2005. [Online]. Available: citeseer.ist.psu.edu/article/biswas05opportunistic.html

[19] H. Fler, J. Widmer, M. Ksemann, M. Mauve, and H. Hartenstein, "Contention-based forwarding for mobile ad hoc networks." [Online] Available: citeseer.ist.psu.edu/693695.html

[20] A. Festag and et al., "Fleetnet: Bringing car-to-car communication into the real world," in Proceedings of the 11th World Congress on ITS, Oct., 2004. 